\section{The Role of Magnetic Resonance Imaging in Diagnosis and Management of Breast Cancer}

www.tcrt.org

A review of the literature on the current applications of breast magnetic resonance imaging (MRI) indications, their rationale and their place in diagnosis and management of breast cancer was given. Contrast-enhanced breast MRI is developing as a valuable adjunct to mammography and sonography. Its high sensitivity for invasive breast cancer establishes its superiority in evaluation of multifocality/multicentricity, tumor response to neoadjuvant chemotherapy, detection of recurrence, and staging. Emerging applications include spectroscopy, usage of new contrast agents, and MRI-guided interventions, including noninvasive treatment of breast cancer. Its potential benefit in screening high-risk women has yet to be established with prospective studies, particularly with regard to false positive results.

Key words: Breast cancer, Magnetic resonance imaging, MRI-guided intervention, Cancer screening.

\section{Introduction}

It has been more than 30 years since magnetic resonance imaging (MRI) was introduced into clinical practice. At the beginning it was used mostly in neuroradiology and musculoskeletal radiology, but over the years its use expanded to other organ systems. Increasing field strengths, development of faster and stronger coils, a wide assortment of dedicated coils, increasing numbers and availability of MRI units (at least in the Western world) helped to establish MRI as the preferred modality for many indications.

Breast MRI was first introduced in the 1980's when several authors described their initial experiences (1-3). It was soon realized that only contrast enhanced (CE) breast MRI had sufficient potential for detection of lesions not seen by mammography and ultrasound, and for non-invasive characterization of breast lesions (4).

Like every imaging technique, breast MRI has its pros and cons. One obvious advantage is the absence of ionizing radiation. There is no need for excessive compression of the breasts during imaging; therefore, most authors do not use compression at all (5). Contrast materials, chelates of gadolinium, used in MRI
Dag Pavic, M.D.*

Marcia A. Koomen, M.D. Cherie M. Kuzmiak, D.O. Y. H. Lee, M.D., Ph.D. Etta D. Pisano, M.D.

\author{
Department of Radiology \\ University of North Carolina at Chapel Hill \\ Medical School \\ CB 7510, 101 Manning Dr. \\ Chapel Hill, NC 27599 USA
}

Abbreviations: MRI, Magnetic resonance imaging; CE, Contrast enhanced; BCS, Breast conserving surgery; DCIS, Ductal carcinoma in situ; SI, Signal intensity; ROI, Region of interest; SNR, Signal-to-noise ratio; BI-RADS, Breast imaging reporting and data system; ACR, American College of Radiology; PACS, Picture archiving and communication system; US, Ultrasound; ${ }^{1} \mathrm{H}$ MRS, Proton magnetic resonance imaging spectroscopy; USPIO, Ultrasmall superparamagnetic iron oxide; Gd-DTPA, Gadolinium diethylenetriamine pentaacetate; FUS, Focused ultrasound; MRS, Magnetic resonance spectroscopy.

\author{
* Corresponding Author: \\ Dag Pavic, M.D. \\ Email: Dag_Pavic@med.unc.edu
}


have significantly lower potential for developing serious allergic reactions than iodine based contrast materials (6-9). MRI, as a multiplanar technique, enables excellent visualization and three-dimensional orientation, with absence of overlapping structures and summation shadows which might obscure a lesion. In addition it has a very high sensitivity (89\%-100\%) for invasive breast cancer (10-12). Most of the indications for breast MRI are based on its high sensitivity.

On the other hand, the main disadvantage of MRI is low specificity $(37 \%-95 \%)$ for breast cancer $(10,13,14)$. The variable specificity is most likely due to wide variations in MRI units, with different field strengths, different coils, proprietary imaging sequences and different post-processing capabilities which make standardization of breast imaging protocols difficult. Besides the non-standardized imaging protocols, patient selection criteria, interpretation criteria and histologic variability of benign and malignant lesions probably also account for the variable specificity $(10,13-15)$.

Claustrophobic reactions, morbid obesity and metallic objects within a patient's body which make the patient ineligible for MRI examination are some of the disadvantages common to all MRI examinations. The MRI examination remains expensive.

In this article an overview of the current status of breast MRI, the indications and the rationale for use of MRI in the diagnosis and management of breast cancer will be given.

\section{Indications}

Breast MRI is intended to non-invasively characterize lesions previously seen on mammography or ultrasound and to detect and characterize those lesions which were not detected with those methods. In addition, MRI-guided interventions, biopsy and preoperative needle localization are being used in further management for those lesions shown with MRI only.

Today, CE breast MRI is being generally accepted for certain indications $(5,16-18)$ :

1. Pre-treatment staging in women with known breast cancer who have dense or heterogeneously dense breasts (BI-RADS a categories 3 and 4) in order to exclude multifocal, multicentric or contralateral breast cancer prior to breast conserving surgery (BCS),

2. Evaluation of the effect of neoadjuvant chemotherapy,

3. Evaluation of the breast after BCS to distinguish between scar and recurrence,

4. Evaluation of the breasts in patients with carcinoma of unknown primary who present with positive

a BI-RADS, Breast Imaging Reporting and Data System. axillary lymph nodes and negative mammography and breast ultrasound,

5. Evaluation of breast implants,

6. Further assessment of problematic mammography and sonography findings in selected high-risk patients.

Due to its high sensitivity for invasive cancer, the possibility of using CE breast MRI for screening in selected subgroups of high-risk women has been investigated. However, the cost and time demands of MRI examination render it unlikely to be widely accepted as a general population screening tool.

\section{Breast MRI Technique}

Non-standardized examination protocols and reporting terminology probably represent the biggest obstacles for wide acceptance of breast MRI (17). However, there are some aspects of the MRI exam which seem to be common to all investigators. First, there should be recent mammograms and, if possible, breast ultrasound available, because comparison of findings obtained with different modalities often can improve diagnostic accuracy. Breast MRI is best performed in the second week of the menstrual cycle in pre-menopausal women and 2-3 months after the cessation of hormone replacement therapy in post-menopausal women $(5,19)$. The patient lies prone in the bore of the MRI unit and has an indwelling intravenous catheter inserted for paramagnetic gadolinium-based contrast injection during the examination. A dedicated bilateral surface breast coil is required for good quality examinations (5).

There are many different protocols published in the literature, partly due to the fact that different MRI units have different capabilities. However, most of the protocols recommend scanning both breasts with $\mathrm{T} 2$ and T1-weighted sequences. Since there can be a significant amount of adipose tissue within breasts, which would produce high signal, fat-suppression sequences are usually being used. Differences exist in slice orientations, and all possible combinations of sagittal, transverse or coronal scans have been reported. Scan thickness varies as well, and is usually 2-4 $\mathrm{mm}$ with no interslice gaps. While most authors obtain preand post-contrast T1-weighted scans, there is wide variety in timing and number of post-contrast series. Most authors use a standard dose of $0.1 \mathrm{mmol} / \mathrm{kg}$ of gadolinium-based paramagnetic contrast for magnetic field strengths 1.0-1.5 T, while for $0.5 \mathrm{~T}$ some recommend using a dosage of 1.5 or 2 times more (5). Very good safety and tolerance profiles of currently used paramagnetic contrast agents have been known since the 1990's (6), so fear of contrast reactions, which are still possible, is not a significant issue.

Post-processing should be considered a part of the examination. Most authors obtain subtraction of pre-contrast images 
from at least one of the post-contrast sets on a pixel-for-pixel basis. Therefore, to achieve high diagnostic quality, it is of utmost importance that the patient not move during the examination. Some authors make an additional single image of the breast(s) using a maximum intensity projection algorithm on the subtracted set, to facilitate quick detection of unsuspected lesions $(5,20)$. For each enhancing lesion, a graph showing its signal intensity (SI) over time on postcontrast scans is then produced. This is usually automatically done, after the examiner positions the region of interest (ROI) marker over the lesion (5).

Recently, an international multicentric investigation on CE breast MRI led by Heywang-Kobrunner was reported (10). It was "initiated in order to allow a more objective assessment of CE breast MRI and to make a first step towards an improved standardization of MRI interpretation". They used a 3D FLASH T1 sequence in coronal orientation, encompassing both breasts, with $2.5 \mathrm{~mm}$ slice thickness. Since there were $1.0 \mathrm{~T}$ and $1.5 \mathrm{~T}$ MRI units at participating sites, particular scanning parameters, i.e., matrix resolution, TR and TE, differed to achieve uniform temporal resolution (87s per scan). For contrast enhancement, they used a 0.2 $\mathrm{mmol} / \mathrm{kg}$ of Gd-DTPA (gadopentetate dimeglumine) bolus with a subsequent saline flush. One could speculate that this particular dose was used to facilitate enhancement seen by 1.0 T MRI units, since SI and signal-to-noise ratio (SNR) are related to magnetic field strength. One pre-contrast series followed by five consecutive post-contrast series were obtained, with the first post-contrast acquisition and contrast injection starting simultaneously. Therefore, the last postcontrast scan was performed 5 min after contrast injection. For all enhancing lesions, a SI/time curve was produced.

\section{Interpretation}

Detection and characterization of a breast lesion on MRI is based on its morphology and the pattern of contrast enhancement. Invasive tumors typically show strong enhancement on the first post-contrast scans, followed by rapid wash-out on subsequent series. This enhancement reflects tumor angiogenesis, since malignancies tend to be highly vascular and tumor blood vessels are up to eight times more permeable than non-tumor vessels (21-23). In addition, there is increased interstitial space, which functions as an additional volume where contrast agent can distribute (24-27). There are also arterio-venous shunting and increased interstitial pressure within the tumor, which might be responsible for the wash-out phenomenon usually seen in malignant lesions (28-30).

There have been many interpretation criteria reported $(14,31$ $34)$. None of them has become widely accepted, partly due to varying MRI techniques employed throughout the world (13).

In the 1990's Fischer et al. introduced a combined morphologic-dynamic score system with five criteria: shape, border, initial signal increase, post-initial signal behavior, and contrast material distribution within the mass (see Table I) (31). Total score, which can range from 0-8 points, can then be translated to BI-RADS categories: 0 - negative; 1,2 benign; 3 - probably benign; 4, 5 - suspicious; 6, 7, 8 - highly suggestive for malignancy, and appropriate action should be undertaken. This system has been tested by several authors and seems to be useful in assessing the probability of malignancy of a breast mass (14). Fischer et al. (31) reported sensitivity of $92.6 \%$ and specificity of $65 \%$ in a group of 463 patients. In a group of 238 patients Baum et al. (14) reported sensitivity for malignancy of $92 \%$ and specificity of $92 \%$. Excluding the cases of ductal carcinoma in situ (DCIS) specificity would increase to $95 \%$, the highest specificity published to date.

Another effort to overcome "the lack of consensus in describing architectural features and/or kinetic data" which "results in major problems in consolidating data from breast MRI studies, evaluating the applicability of any one technique and communicating findings and results" was initiated in 1998, when an International Working Group on Breast MRI was funded "to expedite the clinical implementation and widespread dissemination of breast MRI" $(13,35)$. As part of this effort, a breast MRI lexicon (ACR ${ }^{\mathrm{b}}$ BI-RADS MRI) was developed, tested, and published in 2003 to

b ACR, American College of Radiology.

Table I

The morphologic-dynamic score system for evaluation of masses on CE breast MRI (adapted from Fischer, et al. (31)).

\begin{tabular}{lccc}
\hline \multicolumn{1}{c}{ Features of the mass } & $\mathbf{0}$ & $\mathbf{1}$ & $\mathbf{2}$ \\
\hline Shape & round, oval, lobulated & linear, dendritic, stellate & - \\
Margins & well-defined & ill-defined & - \\
Enhancement pattern & homogeneous & inhomogeneous & rim enhancement \\
Initial enhancement* & low & moderate & high \\
Enhancement over time $^{* *}$ & continuous increase & plateau & wash-out \\
\hline
\end{tabular}

* Peak signal intensity (SI) measured 1-3 min after contrast injection relative to pre-contrast SI: under $50 \%$, within $50-100 \%$, above $100 \%$. ${ }^{* *}$ SI at 3-8 min after contrast injection relative to peak SI: above $10 \%$, within $+/-10 \%$, under $-10 \%$. 
"encompass the reporting of breast MRI scanning technique, lesion architecture, and ROI kinetic/time-intensity curve interpretation" (13). It includes descriptors which were thought to be important for lesion diagnosis and interpretation. The international experts who participated in its development expect it "to change with advances in morphologic and dynamic imaging techniques" (13).

While development of an ACR breast MRI lexicon should be welcomed as a great step towards the much needed standardization in describing findings of breast MRI, it does not actually include a standardized examination protocol. However, on its website, in the ACR's 2004 Guidelines and Standards, there is a Resolution 11: ACR Practice Guidelines for the Performance of Magnetic Resonance Imaging of the Breast, which lists current indications and rather broad specifications of examination with technical guidance and documentation requirements, but no standardized protocols (36).

The aforementioned international group leaded by HeywangKobrunner (10) also addressed the problem of standardization of breast MRI technique and interpretation. They found early enhancement and wash-out parameters to be the most valuable predictors of malignancy. Different weighting of false-negative vs. false-positive calls allowed formulation of a statistically based interpretation scheme yielding optimized rules for the highest possible sensitivity on different specificity levels. With low $(30 \%)$, moderate $(50 \%)$ or high (64$71 \%$ ) specificity they achieved sensitivities of $98 \%, 97 \%$ or $96 \%$, respectively on $1.0 \mathrm{~T}$ MRI units, and $96 \%, 93 \%$ and $86 \%$ on $1.5 \mathrm{~T}$ MRI units. Interestingly, their results showed lower accuracy for the higher field strength, which was explained by the authors: "As confirmed by retrospective in vitro test, the pulse sequence used at $1.5 \mathrm{~T}$ is less sensitive to $\mathrm{T} 1$ changes than the pulse sequence used at 1.0 T. An adaptation of the sensitivity to $\mathrm{T} 1$ changes can be reached by using a higher flip angle at 1.5 T." This observation clearly illustrates the fact that the imaging parameters, as a basic and hence very important component of any imaging protocol, cannot be simply translated from one MRI unit to another, even when they come from the same vendor. One of the interesting results of this study was the fact that no morphological parameter was ever selected as an important predictor in lesion classification. The protocol used, with field of view (FOV) of $160 \times 320 \mathrm{~mm}, 112 \times 256$ matrix and $2.5 \mathrm{~mm}$ slice thickness, resulted in pixel size of $1.43 \times 1.25 \mathrm{~mm}^{2}$ and voxel volume of $4.46 \mathrm{~mm}^{3}$. Therefore, one can speculate that their results were predisposed by using low spatial resolution and that with the use of denser matrix, morphological parameters might have had increased importance.

This opinion is supported by at least two papers. Vomweg et al. (37) compared T1-weighted 3D-FLASH sequences in a 1.5 -T scanner $(512 \times 256$ pixel matrix at high resolution; 256 $\times 128$ pixels at low resolution sequences, 72 slices, $1.7-\mathrm{mm}$ slice thickness, TR $8.8 \mathrm{~ms}$, TE $4.5 \mathrm{~ms}$, flip angle 25 degrees) and concluded that, "This protocol at the charge of slightly enlarged time for measurement offers an elegant way to improve analysis of architectural features in breast MRI." In a retrospective review of 100 consecutive, solitary, MRIonly-detected breast lesions, which were surgically excised after MRI-guided needle localization, Liberman et al. found that spiculated margins, rim enhancement and irregular shape were the strongest predictors of malignancy for masses (only spiculated margin reached statistical significance), while segmental, clumped linear and clumped ductal enhancement had the highest positive predictive values in non-mass lesions (38). Their protocol included separate post-contrast imaging for each breast with sagittal $2 \mathrm{~mm}$ thick sections, no gap, FOV 16-18 cm, matrix $256 \times 192$. Calculated from this data, they had a pixel size of approx. 0.7 $\times 0.9 \mathrm{~mm}^{2}$ and voxels of approx. $1.18 \mathrm{~mm}^{3}$. Therefore, they obtained MRI signal from 4 times smaller tissue volumes than the previously mentioned group, and were able to achieve better visualization of lesion borders. In this study, there were no kinetic post-contrast curves obtained, but the dynamics of contrast enhancement was qualitatively assessed on three consecutive post-contrast series in 78 patients. The authors found that visually assessed kinetic features were not significant predictors of malignancy. Most of the lesions were assessed as having a plateau pattern.

On the contrary, another group of American authors reported, "The addition of qualitative classification of the time-SI curve to an architectural interpretation model results in significant improvement in model performance as measured by the area under the ROC curve" (39). They referred to the previously described interpretation model based on architectural features of suspicious breast MRI findings (33). Interestingly, these authors found that, in the series of 100 enhancing lesions, "quantitative kinetic features demonstrated large standard deviations, however, and they did not appear to represent valuable features for use in lesion classification." They explained these findings with limited temporal resolution and their inability to perform more sophisticated modeling of the kinetic data. The same group published another paper, in which they updated the original model and, using only morphologic criteria, reached sensitivity of $96 \%$ and specificity of $78 \%$ (11).

When one thinks of criteria for differentiating three enhancement patterns, i.e., wash-out, plateau and continuous increase, it is clear that it could be very difficult to qualitatively assess some cases. The plateau pattern is defined as the quantitative change within $\pm 10 \%$ in SI over time. A difference of, for example, $+15 \%$ can be easily shown with direct measurement on the workstation using an ROI placed over the enhancing lesion. Qualitative assessment of that 
case might fall into two categories, plateau or sustained increase, which might reduce accuracy. Even the ROI measurements have their inherent limitations due to variable relation in size of the lesion and the ROIs, and interoperator variability in the selection of ROI within the lesion.

Another issue is the available display medium for assessing contrast uptake. Most European authors have access to a workstation for post-processing or are actually physically present at the MRI console during the exam, so they are able to place the ROI over the enhancing mass and obtain dynamic SI curves. In the United States, the radiologist is rarely present during the exam and, in most cases, reads the images from a PACS ${ }^{\mathrm{c}}$ station. This leaves American authors dependent on features of their particular PACS, that is whether the software available to them is capable of performing dynamic SI curves or not. However, the above mentioned observation is not the rule, as confirmed by the paper published by Schnall et al. (39).

One interesting consideration was addressed in a previously mentioned paper by Liberman et al. [C13]. While mammographically smooth margins of a mass lesion are associated with low likelihood of malignancy, up to $2 \%$ (40), this feature might not be readily applied on MRI imaging. The authors mention that there is a selection bias, since the patients undergoing CE breast MRI are at higher risk than the general population. Secondly, the appreciated smoothness on MRI images is related to the spatial resolution and, to a lesser extent, window and level settings (41). Finally, there are different histologic correlates of the perceived border. In mammography the margin is the interface between the mass and the adjacent parenchyma, while in CE breast MRI, the margin is the interface between the area of vascularity and the surrounding tissue $(26,27)$.

\section{Preoperative Evaluation of Breast Cancer Patients}

Preoperative MRI of the breasts has been proven to be the most sensitive imaging modality in the detection of multifocal, multicentric and contralateral breast cancer (Figure 1). Therefore, MRI is increasingly being used in preoperative evaluation of the extent of disease in breast cancer patients in order to plan appropriate treatment (42-44). It has been shown to have a significant impact on the choice of treatment $(31,45,46)$ and outcome (20).

Hlawatsch et al. (47) compared the diagnostic value of whole-breast sonography and MRI as adjuncts to mammography in preoperative evaluation of a group of 104 women with mammographic findings highly suggestive for malignancy. Authors found that: "Use of sonography benefited 13

c PACS, Picture Archiving and Communication System.
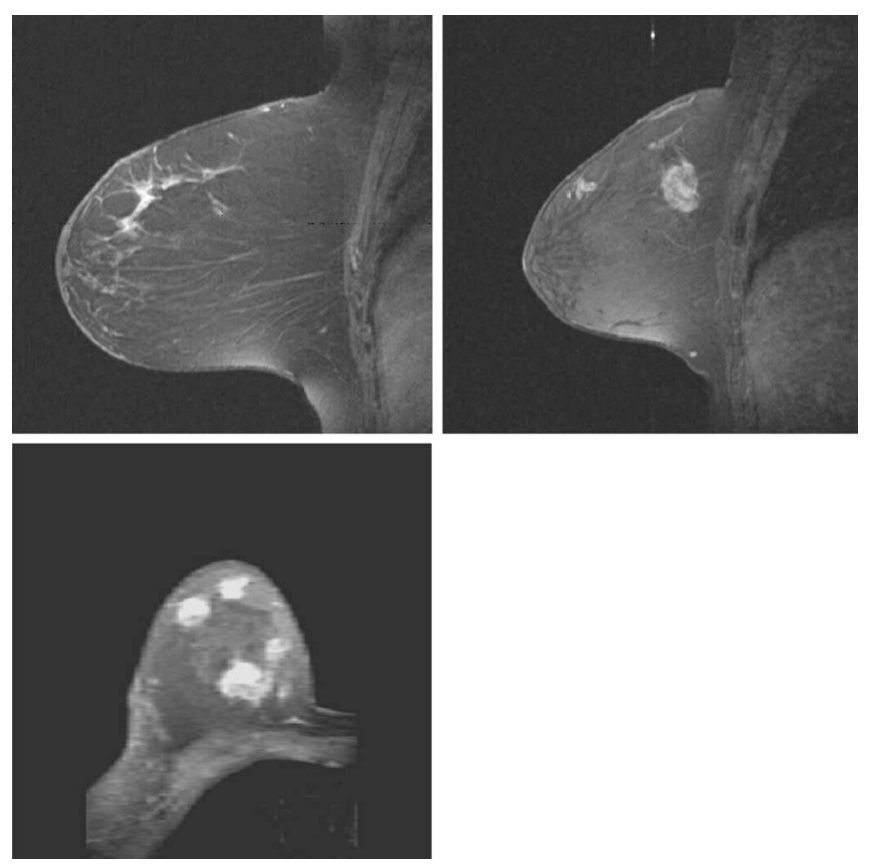

Figure 1: Multifocal and multicentric invasive ductal carcinoma in two different patients. A, Sagittal T1-weighted postcontrast image (3D FLASH spoiled gradient echo, TE $4 \mathrm{~ms} / \mathrm{TR} 30 \mathrm{~ms}$, $3 \mathrm{~mm}$ slice thickness) with two foci of contrast enhancement and architectural distortion in anterior/superior aspect of the breast within the same quadrant, corresponding to multifocal carcinoma. B, Different patient: Sagittal T1-weighted postcontrast image with two separated, irregular, enhancing masses corresponding to carcinomas. C, The same patient as in (B), reformatted transversal Maximum Intensity Projection picture of the same breast with 5 enhancing masses visible in different quadrants, corresponding to multicentric carcinoma.

patients and produced two studies with false-positive findings. Use of MRI imaging benefited seven patients and produced eight studies with false-positive findings." They concluded, "Although MRI imaging is most sensitive for the detection of small tumors, routine preoperative MRI imaging appears to be unnecessary for most patients if a combination of mammography and whole-breast sonography is used. Additional MRI imaging can be restricted to problematic cases in women with dense breast parenchyma."

In a study of 463 patients with 405 malignant lesions, Fischer et al. (31) found multifocality in 30 of 42 patients, multicentricity in 24 of 50 patients, and additional contralateral carcinomas in 15 of 19 patients through the use of MRI only. Due to the MRI findings, therapy was changed correctly in 66 patients (14.3\%) and unnecessary open biopsy was performed in 16 patients (3.5\%). The authors stated that dense or heterogeneously dense breasts were "the only mammographic or US finding to help define a subgroup of patients with multifocal or multicentric disease seen with MRI imaging alone" and concluded that "MRI imaging may reveal unsuspected multifocal, multicentric, or contralateral breast carcinoma and result in therapy changes." 
Tillman et al. (45) evaluated the impact of MRI on the clinical management of 207 early-stage ( 0 - II) breast cancer patients with 212 cancers. They found that the MRI findings affected clinical management in 43 cases. Based on the pathology findings and the overall clinical course, "breast MRI was judged to have had a strongly favorable effect on management in 18 cases (8\%), a somewhat favorable effect in six cases (3\%), an uncertain effect in five cases (2\%), a somewhat unfavorable effect in 11 cases (5\%), and a strongly unfavorable effect in three cases (1\%)." The authors observed that due to the presence of a learning curve for interpreting radiologists, early cases in the study were more likely to be affected by breast MRI and that these effects were more likely to be unfavorable than in later practice. They concluded that "Breast MRI alters the clinical management for a sizeable fraction of women with early-stage breast cancer and appears to offer clinically useful information for determining optimal local treatment."

Liberman et al. (48) evaluated 223 examinations of the asymptomatic, mammographically normal contralateral breast in women whose breast cancer was diagnosed within 6 months preceding MRI imaging. They found that a breast biopsy was performed for 72 contralateral lesions in 61 patients. Thirteen cancers were found in $12(20 \%)$ women who underwent contralateral breast biopsy. Highrisk lesions were found in $18(30 \%)$ patients. However, the authors did not give any conclusion regarding the benefit of detecting early contralateral cancers.

Fischer et al. (20) in their retrospective study evaluated the benefit of preoperative MRI for patients with breast cancer in a group of 346 women. The subgroup of 121 women with preoperative MRI was compared to 225 patients without preoperative MRI. They found a statistically significant reduction in the in-breast tumor recurrence rate $(1.2 \%$ compared to $6.8 \%)$, and the occurrence of contralateral cancer $(1.7 \%$ vs. $4 \%$ ) during the mean follow-up period of 40 months. Based on these results, the authors recommended preoperative MRI of the breasts in patients with pathologically verified breast cancer for local staging.

Hata et al. (49) compared MRI with mammography and sonography in preoperative evaluation of 183 breast cancer patients. They concluded that "MRI can diagnose breast cancer as accurately as ultrasound (US) and more accurately than mammography." As their results showed that "MRI can detect intraductal spread more accurately than the other two methods", they also stated that "MRI appears to be indispensable in breast-conserving surgery to minimize local recurrence."

The literature includes different opinions $(50,51)$, where authors underline the need for prospective evaluation of the potential benefit of preoperative MRI evaluation.
Several questions should be asked here. What is the relevance of multifocal/multicentric foci of disease seen on breast MRI? Does their presence influence patients' final outcomes? A focus is defined as an enhancing area under 5 $\mathrm{mm}$ in size which is too small to be characterized and described as a mass. One can speculate that those small areas will be effectively eradicated by postoperative radiation of the breast. Even without radiation, most of the distant microscopic foci found on mastectomy specimens tend not to turn to invasive cancers $(52,53)$.

However, intra-operative radiation therapy for early cancers has been investigated for over a decade. Instead of fractionated postoperative irradiation of the breast over the course of 4 to 6 weeks, single intraoperative radiation dose is applied to the tumor bed. In these women, distant foci of disease might not be effectively treated with radiation and might pose a threat for cancer relapse $(51,54)$. As of now, there is no definite answer $(55,56)$.

\section{Lymph Node Status}

Axillary lymph node involvement is the most important prognostic variable for breast cancer survival and is considered one of the key factors in treatment planning $(57,58)$. Sentinel lymph node biopsy remains the gold standard in assessment of lymph node involvement. What are the options to evaluate nodes non-invasively?

Axillary nodes are often seen on mammograms, but little can be said from their mammographic appearance. Furthermore, some lymph nodes might be located too high to be included on the mammogram. Ultrasound of the axilla in patients with a suspicious palpable breast mass or biopsy-proven cancer is often ordered in an attempt to evaluate possible lymph node involvement. Only level 1 axillary lymph nodes are readily visible by ultrasound. However, palpable level 2 nodes are almost always assessable sonographically. Sonographic characteristics of a lymph node might suggest its involvement if there is gross metastasis with or without new transcapsular blood vessels (59). For microinvasion, US gives no information.

$\mathrm{CE}$ breast MRI might enable non-invasive assessment of lymph node status. Luciani et al. (60) used a standard bilateral breast coil combined with a small axillary surface coil in order to depict axillary lymph nodes in 16 breast cancer patients. Investigators found that a short axis dimension greater than $5 \mathrm{~mm}$, irregular contours, high signal intensity on inversion recovery $\mathrm{T} 2$-weighted images, and intense postcontrast enhancement all represent strong predictors for the presence of axillary lymph node involvement. The mean increase in examination time was 14 minutes. One can only speculate whether the metastases in the involved lymph 
nodes were detectable by ultrasound, and what was their size, since those data were not published in the paper.

Yeung et al. (61) also used a combination of standard breast coil and smaller superficial coil to prospectively evaluate suspicious breast lesions and ipsilateral axillae in 43 patients. They described proton MRI spectroscopy $\left({ }^{1} \mathrm{H}\right.$ MRS) findings in 35 of them, where they found a choline peak confirming the presence of lymph node metastasis in 14 patients. Taking the pathology results on the subsequent lymph node dissection as the gold standard, they achieved sensitivity of $69 \%$, specificity of $100 \%$ and accuracy of $78 \%$.

Kvistad et al. (57) evaluated preoperative dynamic CE MRI for assessment of axillary lymph nodes in a group of 65 patients with invasive breast cancer treated with axillary lymph node dissection. They found that "When using a signal intensity increase in the lymph nodes of $>100 \%$ during the first post-contrast image as a threshold for malignancy, 57 of 65 patients were correctly classified (sensitivity $83 \%$, specificity $90 \%$, accuracy $88 \%$ ). These results were not improved when lymph node size and morphology were used as additional criteria."

New methods for nodal staging are currently being investigated. Thus Michel et al. (62) evaluated ultrasmall superparamagnetic iron oxide (USPIO), a lymph node targeting agent, in axillary lymph node staging. In 20 women who were scheduled for surgery, they performed gadoliniumenhanced MRI imaging 24-36 hours after the start of intravenous slow-drip infusion of USPIO. They reported a sensitivity of $73-83 \%$ and a specificity of $96-97 \%$ in evaluation of 405 identified lymph nodes. They stated that "USPIO as the intravascular contrast agent could not replace gadolinium for assessment of the primary tumor; however, no clinically relevant interaction was seen."

The recent report of Yamagami et al. (63) describes a method for preoperative MRI-guided axillary lymph node biopsy. This group used an open MRI unit for preoperative sentinel node needle biopsy in nine patients with nonpalpable axillary nodes. In seven of nine cases, a specimen sufficient for pathological evaluation was obtained with $18 \mathrm{G}$ biopsy needles. The authors concluded that "This experience indicates the possibility of a precise diagnosis of whether the sentinel node in breast cancer is benign or malignant without surgery." The main limitations of this method are the paucity of open MRI units, cost of MR time and possibility of sampling error.

Given the importance of the axillary node involvement, histologic diagnosis is likely to remain the only definitive method of assessment.

\section{Evaluation of Neoadjuvant Chemotherapy Response}

Breast MRI has been used to evaluate the response of breast cancer to chemotherapy $(27,64-68)$ (Figure 2). .
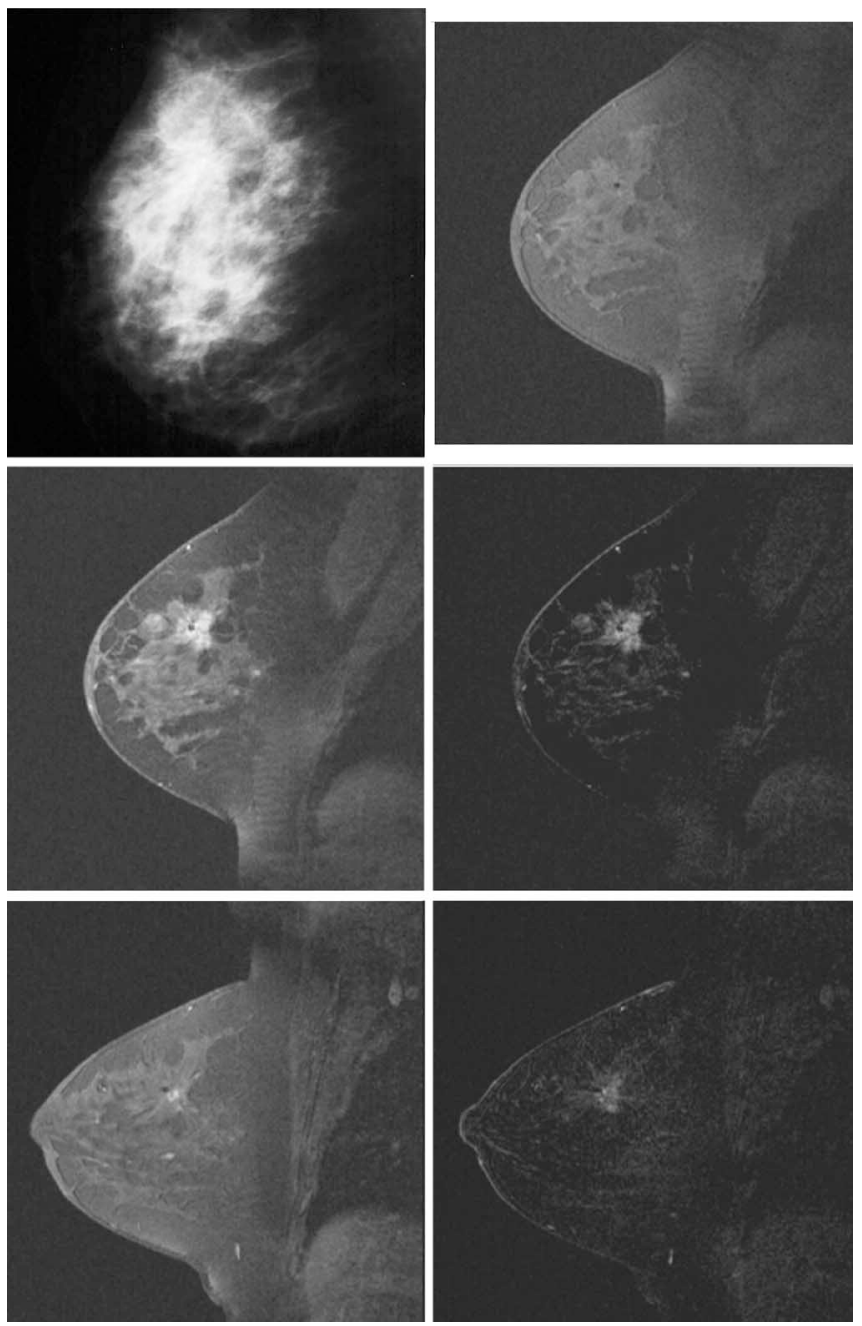

Figure 2: Imaging of invasive ductal carcinoma prior to $\mathbf{A}, \mathbf{B}, \mathbf{C}, \mathbf{D}$ and after $\mathbf{E}, \mathbf{F}$ chemotherapy. A, medio-lateral oblique mammogram with spiculated mass and architectural distortion in superior aspect of the breast. Oval mass anterior to it corresponds to fibroadenoma. B, Pre-contrast sagittal T1-weighted image (3D FLASH spoiled gradient echo, $4 \mathrm{~ms} / 30 \mathrm{~ms}, 3 \mathrm{~mm}$ slice thickness). A small black dot in the center of subtle architectural distortion corresponds to a signal void due to a $24 \mathrm{~K}$ golden marker clip which was placed in approximate center of the cancer during the ultrasound-guided core biopsy. C, The same slice after contrast administration depicts cancer's post-contrast enhancement. D, Subtracted image of the same slice improves visualization of the cancer. A small enhancing area anterior to it corresponds to fibroadenoma. E, Post-contrast sagittal T1-weighted image at approximately the same level, acquired after the course of chemotherapy, shows significant decrease in intensity and size of post-contrast enhancement, indicating shrinkage of the cancer. F, Subtracted image of the same slice. Note persistent architectural distortion.

Riebe et al. (64) performed multiple MRI examinations in 13 patients before, during and after chemotherapy. All patients underwent surgery, and MRI findings were corre- 
lated with pathology. They observed flattening of the GdDTPA uptake curve after the first cycle of chemotherapy or even complete absence of contrast uptake after the fourth cycle in the patients who were classified as responders. However, the authors stated that "the effect of cytotoxic agents on the dynamics of contrast medium uptake in primary breast carcinoma or recurrent disease is not known." They found that "the change in Gd-DTPA uptake behavior led to an underestimation of the extent of tumor in two patients and false negative findings in four patients" and concluded that breast MRI can provide evidence of a chemotherapeutic response, but that no reliable statements regarding residual tumor volume can be made.

Londero et al. (66) compared accuracy of mammography, US and MRI in identifying residual disease after neoadjuvant chemotherapy in 15 patients who ultimately underwent surgery. They concluded that "MRI assesses response to neoadjuvant chemotherapy better than traditional methods of physical examination and mammography." They also observed that sonography performed after MRI improved diagnostic accuracy in evaluation of uncertain foci of multifocal disease seen on MRI images with an increase of diagnostic accuracy from 73 to $84.5 \%$. Two false-negative MRI results were proven to represent microfoci of DCIS and invasive lobular carcinoma on pathology. This is consistent with reported low sensitivity of MRI for these entities.

Rosen et al. (69) also demonstrated that "MRI can show residual malignancy after neoadjuvant chemotherapy better than physical examination, particularly in patients who have not had a complete clinical response to therapy." In a group of 21 women they found that "MRI underestimated the extent of residual tumor in two patients by more than $1 \mathrm{~cm}$ (including one false-negative examination), was within $1 \mathrm{~cm}$ in 12 of 21 patients, and overestimated tumor extent by more than $1 \mathrm{~cm}$ in seven of 21 patients."

Martincich et al. evaluated CE breast MRI as a method to predict the effect of chemotherapy in a group of 30 patients (70) with breast cancer larger than $3 \mathrm{~cm}$. They found that "a greater than $65 \%$ reduction in the tumor volume and a reduction in the early enhancement ratio after two cycles of primary chemotherapy were associated with a major histopathological response," i.e., small clusters of dispersed residual cancer cells or no residual viable cancer cell in pathologic specimens. The authors concluded that "dynamic CE-MRI has the potential to provide functional parameters that could be integrated to optimize neoadjuvant chemotherapy strategies."

\section{Evaluation of Postlumpectomy Breast}

Fisher et al. (55) evaluated a group of 1039 patients 15 years after lumpectomy. They found local recurrence of invasive breast cancer in $36 \%$ of the women who did not undergo irradiation, and in $12 \%$ of those who had radiation after lumpectomy. For patients with DCIS, recurrence rates at an 8-year follow-up interval were $31 \%$ and $13 \%$, in subgroups with and without radiation, respectively (71).

Detection of residual disease and accurate assessment of its extent are required for further management of breast cancer patients. If there is a small amount of residual cancer the patient might undergo re-excision prior to radiation. With extensive residual disease, mastectomy is the treatment of choice (72).

Due to its high sensitivity, MRI might represent a method of choice for postoperative evaluation in patients with positive margins and, for women who have not had preoperative MRI, to evaluate for unsuspected foci of malignancy in the ipsi- and/or contralateral breast (73). Post-contrast enhancement due to post-surgical inflammatory changes at the surgery site limits diagnostic accuracy of MRI for residual breast cancer (74). Optimal time interval between surgery and MRI examination is still controversial (73-77).

Frei et al. (74) evaluated the influence of the time interval between lumpectomy and MRI examination on the diagnosis of residual breast cancer in 68 patients. They found that "the time interval between lumpectomy and MRI of the breast had the greatest influence on the specificity and negative predictive value of MRI, increasing progressively over time. A plateau of highest values of $75 \%$ specificity and $86 \%$ negative predictive value was reached at 28 and 35 days after surgery, respectively. Although the sensitivity and positive predictive value showed smaller variations over time, peak values of $95 \%$ sensitivity and $92 \%$ positive predictive value were obtained at 35 and 28 days after surgery, respectively." Therefore, they recommend MRI examination for patients with positive resection margins no earlier than 28 days after initial surgery. This limits its utility since most women would prefer to commence radiation therapy before this interval elapses.

Breast MRI has been increasingly used to evaluate the breast after lumpectomy, to differentiate between scar and recurrent malignancy (78). The discriminating feature for this differentiation is post-contrast enhancement, which should be present in recurrent or residual malignancy and absent in scar tissue. Most authors consider 18 months a time limit for this differentiation (76), since new, i.e., "young" scars can also show post-contrast enhancement, which has been confirmed in one contrast-enhanced ultrasound study, too (79).

Drew et al. (77) investigated the clinical application of breast MRI in screening for local recurrence following BCS in 105 patients in the period 1 to 2 years following treatment, or ear- 
lier if recurrence was suspected. They concluded that "combined clinical examination and mammography are as sensitive as dedicated dynamic MRI of the breast for the detection of locoregional recurrence, but breast MRI is associated with a far greater specificity. Therefore, dedicated dynamic breast MRI should be used when there is clinical or mammographic suspicion of recurrence to confirm or refute its presence."

New contrast agents which might help in differentiating scar from recurrence are under investigation. Thus, Lee $e t$ al. (22) reported that a blood pool contrast agent, SH L $643 \mathrm{~A}$, in an experimental model showed promising results in differentiating the two.

\section{MRI-guided Interventions}

Percutaneous biopsy under stereotactic or sonographic guidance has been shown to be a reliable method for histologic diagnosis of breast lesions. It is faster, cheaper, and, in general, better tolerated than surgery (80).

The lack of reliable methods for non-surgical sampling of solely MRI-detected suspicious breast lesions has hindered the utilization of breast MRI (81). For lesions seen exclusively with MRI, the only option was MRI-guided preoperative needle localization and surgical excision (82, 83). MRI-guided preoperative placement of a localization wire has been reported to be feasible and successful in several reports (84-87).

Any intervention under MRI guidance poses several technical difficulties $(84,88)$. Except with open MRI units, patients have to be moved in and out of the magnet bore repeatedly during the procedure. Lesions located in inner quadrants might be hard to access, since the patient lies prone in a bilateral breast coil. Lesion visibility decreases over time after the injection of contrast due to the transient nature of contrast enhancement. Despite initial experience of free-hand localizing methods $(81,87)$, today most authors use some kind of compression device and have the patient lying prone during the procedure. Different types of compression devices have been developed. Despite differences, they all have to fulfill two requirements: they have to enable moderate compression to immobilize the breast during the procedure, and there should be enough openings over the whole side of the breast to enable insertion of biopsy needle or localization wire through the compression plate in the desired position.

Also, the breast may appear different with no compression, as in diagnostic MRI, and with compression, as used in interventional MRI-guided procedures, which might pose a problem in detection of a lesion during procedures. Postcontrast enhancement might be diminished or absent if the compression is too vigorous (88-90).
For MRI-guided preoperative needle localization of lesions seen exclusively on MRI (Figure 3), confirmation of lesion excision is difficult, if not impossible, since no enhancement is possible ex vivo $(82,84,88)$.
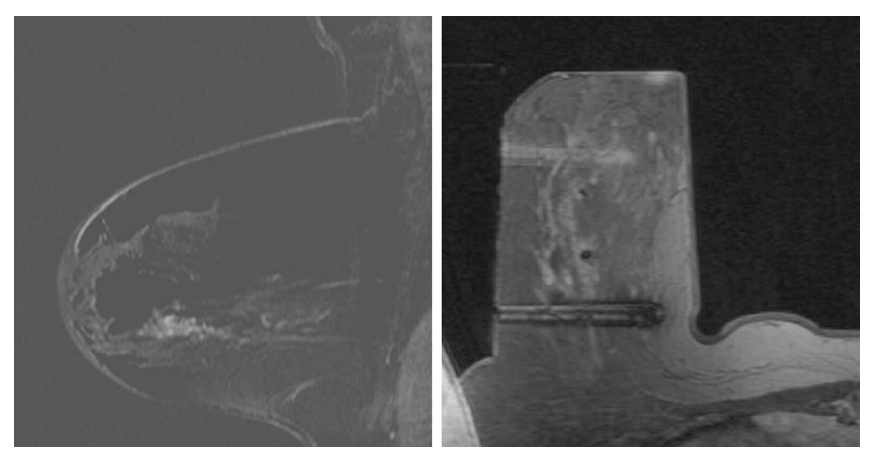

Figure 3: Preoperative MRI-guided needle localization. Pathology revealed lobular carcinoma in situ. A, Subtracted post-contrast sagittal image of the breast showing non-mass-like, clumped, ductal enhancement which was considered suspicious. B, Transverse postcontrast T1 weighted image of the compressed breast acquired during localization procedure, with two needles inserted, bracketing the suspicious area. Note that different appearance of the needles' artifacts is due to fact that they were inserted at different transversal levels. Two punctate areas of signal void between the needles correspond to marker-clips placed during prior percutaneous biopsies.

MRI-guided percutaneous biopsies for breast lesions have been reported in the last decade (91) Initially, MRI-guided core biopsy has been limited by lower sampling accuracy and lack of MRI-compatible biopsy needles. This unfavorable situation has changed in recent years, with several vendors offering MRI-compatible vacuum-assisted biopsy systems.

Kuhl et al. (92) reported results of MRI-guided automated $14 \mathrm{G}$ core needle biopsy of 78 lesions visible at MRI imaging only, in 59 patients. Histologic diagnosis from core biopsy was possible in 77 (99\%) of 78 lesions. In the 59 lesions with established pathologic validation, the diagnostic accuracy was $98 \%$ (58 of 59). The authors concluded that "MRI imaging-guided large-core stereotactic breast biopsy is sufficiently accurate for obtaining histologic proof of lesions visible only at MRI imaging. It can change patient treatment by reducing unnecessary surgical biopsy and can enable onestep surgery for breast cancers."

Perlet et al. (93) reported their results with MRI-guided vacuum biopsy of 206 contrast-enhancing breast lesions. In the 4 cases where biopsy was unsuccessful, the failure was realized during the procedure, thus avoiding false negative results. All other biopsies were successful and accurate diagnosis was established, except for one case of DCIS which was characterized as atypical ductal hyperplasia at the biopsy. The authors concluded that "MRIguided vacuum biopsy allows reliable histological workup of contrast-enhancing small lesions which are not visible by any other modality." 
Liberman et al. (94) reported results of MRI-guided vacuum-assisted biopsy of 27 lesions in 19 women, followed by subsequent immediate localization wire placement and surgical excision. The authors concluded that "MRI-guided vacuum-assisted biopsy is a fast, safe, and accurate alternative to surgical biopsy for breast lesions detected on MRI."

Vacuum-assisted biopsy probes have an important advantage, ability to obtain relatively large sample volumes, which leads to a lower probability of insufficient sampling. Furthermore, it is possible to place a marker clip at the biopsy site. This clip is visible on mammograms and, in case of positive biopsy results, preoperative needle localization can be performed with mammography guidance.

Huber et al. (95) reported a noninvasive MRI-guided focused ultrasound (FUS) for treatment of human breast cancer. They developed a FUS therapy unit, compatible with and guided by clinical $1.5 \mathrm{~T}$ MRI unit. "With interactive target segmentation on MRI, defined volumes could be noninvasively treated in a single session with on-line MRI temperature control. The ultrasound waves were focused through the intact skin and resulted in localized thermal tissue ablation at a maximum temperature of $70{ }^{\circ} \mathrm{C} . "$

The authors concluded, "Immunohistochemistry of the resected specimen demonstrated that FUS homogeneously induced lethal and sublethal tumor damage with consecutive up-regulation of p53 and loss of proliferative activity. This effect was realized without anesthesia and damage to the surrounding healthy tissue or systemic effects. Overall, our results show that therapy of breast cancer is feasible and effective. Thus, MRI-guided FUS may represent a new strategy for the neoadjuvant, adjuvant, or palliative treatment in selected breast cancer patients and in patients with other soft-tissue tumors."

Treatment success and residual tumor can be monitored by CE breast MRI as shown by Gianfelice et al. (96). Of course, use of MRI for guidance of the procedure takes a lot of time, perhaps limiting its availability.

\section{Magnetic Resonance Spectroscopy and Perfusion Imaging}

Magetic resonance spectroscopy (MRS) has been used in clinical practice for evaluation of brain tumors as a method for noninvasive detection of tumor metabolism $(97,98)$. In the late 1990's first attempts to better characterize breast lesions were performed using MRS (99). Compared to normal breast parenchyma, breast cancers show increased levels of phosphocholine and choline. While these tumor-distinctive metabolites are best detected with ${ }^{31} \mathrm{P}$ MRS in vitro, this technique is not readily applicable in vivo due to lower MRI sensitivity in detection of phosphorus-originated signals compared to proton signals, and consequently significantly longer acquisition time $(99,100)$. Therefore, single-voxel ${ }^{1} \mathrm{H}$ MRS has been used in clinical practice, since it adds about ten minutes to the overall examination time for one measurement (99). With increasing availability of higher field strength MRI units in clinical practice [3 T], more suitable for MRS, breast MRS might be expected to become a part of the MRI examination protocol (101).

In several studies, sensitivity of breast single-voxel ${ }^{1} \mathrm{H}$ MRS for detecting breast cancer ranged from $73 \%-92 \%$ with specificity of $71 \%-93 \%(61,102-104)$. According to Tse et al. (105) current technical limitations allow for recommendation of using single-voxel ${ }^{1} \mathrm{H}$ MRS in evaluation of masses $1.5 \mathrm{~cm}$ and bigger in size.

In a recent paper, Jacobs et al. (106) suggested that single slice magnetic resonance spectroscopic imaging has advantages over single-voxel ${ }^{1} \mathrm{H}$ MRS. In their preliminary report of 18 patients, they performed single-slice MRSI (10-mm slice thickness) and then, from MRSI data, reconstructed water, lipid, and choline maps. They concluded that "MRSI measurements of choline are feasible in the human breast, and the SNR for choline was significantly different between benign and malignant lesions. The potential advantages of MRSI over single-voxel spectroscopy include the ability to assess multiple lesions as well as tissue with normal MRI appearance, as well as to perhaps gauge lesion borders and infiltration into surrounding tissue."

Huang et al. (107) recently reported an increase in specificity from $62.5 \%$ to $100 \%$ when single-voxel ${ }^{1} \mathrm{H}$ MRS and T2* perfusion imaging were added to dynamic CE breast MRI. They prospectively evaluated 50 patients with positive mammography findings prior to biopsy. In 28 patients with postcontrast enhancement ${ }^{1} \mathrm{H}$ MRS and T2* perfusion were performed. The authors concluded that "the combined MRI protocol of dynamic CE MRI imaging, ${ }^{1} \mathrm{H}$ MRS spectroscopy, and perfusion MRI imaging has high sensitivity and specificity in the diagnosis of breast cancer."

T2*-weighted first-pass perfusion imaging is based on increased perfusion typical for malignant tumors. Kvistad $e t$ al. (108) described the following technique:

After the first dose of Gadolinium chelate contrast was injected and several postcontrast T1-weighted scans were obtained, a single slice where the contrast enhancing lesion is seen the best is chosen. A gradient-echo $\mathrm{T} 2 *$ sequence is then obtained on that section, with an acquisition time of about $5 \mathrm{sec}$ onds. That sequence is repeated 40 times. After the first 10 repetitions, the second dose of $0.1 \mathrm{mmol} / \mathrm{kg}$ of gadolinium contrast is rapidly injected as a bolus followed by saline flush. The time between the first 
contrast dose, used for T1-weighted imaging, and the second contrast dose, was approximately 15 minutes. On the $\mathrm{T} 2 *$-weighted images, an ROI was positioned in the part of tumor enhancing the most on the T1-weighted images, and a time-SI curve was obtained. The maximum SI loss within the first 30 seconds after contrast bolus injection was calculated as a percentage of the baseline SI values from the 10 pre-contrast images. In their study on 130 patients with breast tumors, they observed different levels of SI loss for benign and malignant lesions. For carcinomas, SI loss was $31 \pm 15 \%$, while for benign lesions it was $9 \pm 7 \%$. When they used a cut-off of $20 \%$ and more of a SI loss as a sign of malignancy, they achieved a sensitivity of $79 \%$ and a specificity of $94 \%$. Four false-positive lesions consisted of 3 fibroadenomas and one papilloma. Three of these lesions were found in premenopausal women, and the fourth patient was on hormone replacement therapy.

\section{Screening in High-risk Women}

Numerous controlled trials have demonstrated that screening mammography leads to a reduction in breast cancer mortality of up to $30 \%$ (109-111). The benefit of screening mammography has generally been accepted, despite the recent controversy ignited by a front-page article in the New York Times $(109,112,113)$. Risk of breast cancer is increased in breast oncogene carriers, women with a personal or a family history of breast cancer, and those with biopsy-proven atypia, lobular carcinoma in situ or radial scar (114). Women with mutations of the BRCA1 and BRCA2 genes have an $85 \%$ lifetime risk of breast cancer (115). Several studies suggest that mammography has a lower sensitivity in these women than in the general population $(116,117)$. Breast cancer in oncogene carriers is more likely to be high-grade and hormone receptor negative, rendering early detection crucial (118).

Until now, management options for high-risk women ranged from close surveillance, with clinical breast examination every six months and annual mammography, to prophylactic bilateral mastectomy $(114,119)$. The high sensitivity of MRI for invasive breast cancer has led to interest in its use as a screening tool in selected subgroups of high-risk patients. MRI screening holds the promise of higher sensitivity, but there is also the burden of false positives, with increased numbers of negative biopsies based on MRI findings, carrying increased morbidity, anxiety, and induced costs $(17,120)$.

Morris et al. (114) investigated the rate and results of biopsy in the first round of breast MRI screening in 364 asymptomatic, high-risk women with normal mammograms.
Based on MRI findings, biopsy was recommended in 64 (17\%), and performed in 59 women. None of these lesions had a mammographic correlate. In 44 of these women, who were sonographically evaluated after the MRI finding, US identified 11 lesions (25\%). Biopsy revealed 16 clinically and mammographically occult cancers in 14 women, $4 \%$ of those who underwent MRI screening. More than half of the cancers detected (57\%) were DCIS. Two cancers had US correlate, one woman had multifocal cancer and one had bilateral cancer detected by MRI. Based on their results, these authors suggest several subgroups of high-risk patients who are most likely to benefit from MRI screening. In declining likelihood of malignancy, these are women with both personal and family history of breast cancer, postlumpectomy patients, and women with a positive family history. However, they concluded that, "Further work, including refinements in technique and interpretation; longterm follow-up; and assessments of sensitivity, specificity, and cost effectiveness, is needed to define the role of breast MRI in screening women at high risk."

The recent report by Kriege et al. (121) on the efficacy of MRI and mammography for screening in 1909 women with a genetic or familial predisposition to breast cancer might open a door for wider utilization of MRI screening in highrisk women. The study was conducted in the Netherlands and encompassed asymptomatic women with a lifetime risk of breast cancer of $15 \%$ or more. Surveillance consisted of a clinical breast examination every six months, and imaging studies annually, mammography and dynamic CE breast MRI. The authors wanted to determine whether MRI screening facilitated the early diagnosis of hereditary breast cancer. The sensitivity for invasive cancer was $79.5 \%$ for MRI, compared to $33.3 \%$ for mammography and $17.9 \%$ for clinical examination. Specificities were $89.8 \%, 95.0 \%$ and $98.1 \%$, respectively. The proportion of invasive tumors $1 \mathrm{~cm}$ and smaller and the incidence of positive axillary lymph nodes was significantly lower in the study group, compared with two different, age-matched control groups. The authors concluded that, "MRI appears to be more sensitive than mammography in detecting tumors in women with an inherited susceptibility to breast cancer." One can argue about the differences in mammography screening in the United States compared to European countries (lower threshold for abnormalities on screening mammograms, which results in significantly higher call-back rates in the United States), and the relatively short follow-up period (median 2.9 years) in this study, but these results remain impressive.

\section{Conclusion}

CE breast MRI is developing as a valuable adjunct to mammography and sonography. Its high sensitivity for invasive breast cancer establishes its superiority in evaluation of multi- 
focality/multicentricity, tumor response to neoadjuvant chemotherapy, detection of recurrence, and staging. Emerging applications include spectroscopy, usage of new contrast agents, and MRI-guided interventions, including noninvasive treatment of breast cancer. Its potential benefit in screening high-risk women has yet to be established with prospective studies, particularly with regard to false positive results.

\section{References}

1. Heywang, S. H., Bassermann, R., Fenzl, G., Nathrath, W., Hahn, D., Beck, R., Krischke, I., and Eiermann, W. MRI of the Breast Histopathologic Correlation. Eur. J. Radiol. 7, 175-182 (1987).

2. El Yousef, S. J. and Duchesneau, R. H. Magnetic Resonance Imaging of the Human Breast: A Phase I Trial. Radiol. Clin. North. Am. 22, 859-868 (1984).

3. Dash, N., Lupetin, A. R., Daffner, R. H., Deeb, Z. L., Sefczek, R. J., and Schapiro, R. L. Magnetic Resonance Imaging in the Diagnosis of Breast Disease. Am. J. Roentgenol. 146, 119-125 (1986).

4. Heywang, S. H., Hahn, D., Schmidt, H., Krischke, I., Eiermann, W., Bassermann, R., and Lissner, J. MR Imaging of the Breast Using Gadolinium-DTPA. J. Comput. Assist. Tomogr. 10, 199-204 (1986).

5. Sardanelli, F., Lozzelli, A., and Fausto, A. MR Imaging of the Breast: Indications, Established Technique, and New Directions. Eur. Radiol. 13 Suppl 3, 28-36 (2003).

6. Niendorf, H. P., Alhassan, A., Geens, V. R., and Clauss, W. Safety Review of Gadopentetate dimeglumine. Extended Clinical Experience after more than Five Million Applications. Invest. Radiol. 29 Suppl 2, S179-182 (1994).

7. Sardanelli, F., Mancardi, G., and Filippi, M. Safety of the Long-time Monthly Triple Dose of a Gd-based Contrast Agent. Eur. Radiol. 13 Suppl 4, L243-244 (2003).

8. Haustein, J., Laniado, M., Niendorf, H. P., Louton, T., Beck, W., Planitzer, J., Schoffel, M., Reiser, M., Kaiser, W., and Schorner, W. Triple-dose Versus Standard-dose Gadopentetate dimeglumine: A Randomized Study in 199 Patients. Radiology 186, 855-860 (1993).

9. Okada, S., Katagiri, K., Kumazaki, T., Yokoyama, H. Safety of Gadolinium Contrast Agent in Hemodialysis Patients. Acta. Radiol. 42, 339-341 (2001).

10. Heywang-Kobrunner, S. H., Bick, U., Bradley, W. G. Jr., Bone, B., Casselman, J., Coulthard, A., Fischer, U., Muller-Schimpfle, M., Oellinger, H., Patt, R., Teubner, J., Friedrich, M., Newstead, G., Holland, R., Schauer, A., Sickles, E. A., Tabar, L., and Waisman, J., Wernecke, K. D. International Investigation of Breast MRI: Results of a Multicentre Study (11 sites) Concerning Diagnostic Parameters for Contrast-Enhanced MRI Based on 519 Histopathologically Correlated Lesions. Eur. Radiol. 11, 531-546 (2001).

11. Nunes, L. W., Schnall, M. D., and Orel, S. G. Update of Breast MR Imaging Architectural Interpretation Model. Radiology 219, 484494 (2001).

12. Ikeda, O., Yamashita, Y., Morishita, S., Kido, T., Kitajima, M., Okamura, K., Fukuda, S., and Takahashi, M. Characterization of Breast Masses by Dynamic Enhanced MR Imaging. A Logistic Regression Analysis. Acta. Radiol. 40, 585-592 (1999).

13. American College of Radiology. Breast Imaging Reporting and Data System Atlas, $4^{\text {th }}$ ed. Reston, VA: American College of Radiology (2003).

14. Baum, F., Fischer, U., Vosshenrich, R., and Grabbe, E. Classification of Hypervascularized Lesions in CE MR Imaging of the Breast. Eur. Radiol. 12, 1087-1092 (2002).

15. Orel, S. G. Differentiating Benign from Malignant Enhancing Lesions Identified at MR Imaging of the Breast: Are Time-Signal Intensity Curves an Accurate Predictor? Radiology 211, 5-7 (1999).
16. Del Maschio, A., De Gaspari, A., and Panizza P. Present Indications for the Use of Breast MRI. J. Exp. Clin. Cancer. Res. 21 Suppl. 3, 55-58 (2002).

17. Lee, C. H. and Weinreb, J. C. The Use of Magnetic Resonance Imaging in Breast Cancer Screening Volume 1, Issue 3, 176-182 (2004).

18. Offodile, R. S., Daniel, B. L., Jeffrey, S. S., Wapnir, I., Dirbas, F. M., and Ikeda, D. M. Magnetic Resonance Imaging of Suspicious Breast Masses Seen on One Mammographic View. Breast J. 10, 416-422 (2004).

19. Pfleiderer, S. O., Sachse, S., Sauner, D., Marx, C., Malich, A., Wurdinger, S., Kaiser, W. A. Changes in Magnetic Resonance Mammography Due to Hormone Replacement Therapy. Breast Cancer Res. 6, R232-238 (2004)

20. Fischer, U., Zachariae, O., Baum, F., Von Heyden, D., Funke, M., and Liersch, T. The Influence of Preoperative MRI of the Breasts on Recurrence Rate in Patients with Breast Cancer. Eur. Radiol. Jul 10 2004 [Epub ahead of print].

21. Turetschek, K., Huber, S., Helbich, T., Floyd, E., Tarlo, K. S., Roberts, T. P., Shames, D. M., Wendland, M. F., and Brasch, R. C. Dynamic MRI Enhanced with Albumin-(Gd-DTPA)30 or Ultrasmall Superparamagnetic Iron Oxide Particles (NC100150 Injection) for the Measurement of Microvessel Permeability in Experimental Breast Tumors. Acad. Radiol. 9 Suppl 1, S112-114 (2002).

22. Lee, J. W., Moon, W. K., Weinmann, H.-J., Kim, S. J., Kim, J. H., Park, S. H., Kim, T. J., Yoon, C. J., Kim, Y. H., Cho, E. Y., Ha, S. W., Kang, W.-S., and Chang, K. H. Contrast-enhanced MR Imaging of Postoperative Scars and VX2 Carcinoma in Rabbits: Comparison of Macromolecular Contrast Agent and Gadopentetate Dimeglumine1. Radiology 229, 132-139 (2003).

23. Furman-Haran, E.., Kelcz, F., and Degani, H. Magnetic Resonance Imaging of Breast Cancer Angiogenesis: A Review. J. Exp. Clin. Cancer Res. 21, 47-54 (2002).

24. Heywang-Kobrunner, S. H. and Beck, R. Contrast-enhanced MRI of the Breast, $2^{\text {nd }}$ edition. Springer-Verlag, Berlin (1995).

25. Ikeda, O., Nishimura, R., Miyayama, H., Yasunaga, T., Ozaki, Y., Tuji, A., and Yamashita, Y. Evaluation of Tumor Angiogenesis Using Dynamic Enhanced Magnetic Resonance Imaging: Comparison of Plasma Vascular Endothelial Growth Factor, Hemodynamic, and Pharmacokinetic Parameters. Acta. Radiol. 45, 446452 (2004).

26. Frouge, C., Guinebretiere, J. M., Contesso, G., Di Paola, R., and Blery, M. Correlation Between Contrast Enhancement in Dynamic Magnetic Resonance Imaging of the Breast and Tumor Angiogenesis. Invest. Radiol. 29, 1043-1049 (1994).

27. Buadu, L. D., Murakami, J., Murayama, S., Hashiguchi, N., Sakai, S., Masuda, K., Toyoshima, S., Kuroki, S., and Ohno, S. Breast Lesions: Correlation of Contrast Medium Enhancement Patterns on MR Images with Histopathologic Findings and Tumor Angiogenesis. Radiology 200, 639-649 (1996).

28. Poptani, H., Bansal, N., Graham, R. A., Mancuso, A., Nelson, D. S., and Glickson, J. D. Detecting Early Response to Cyclophosphamide Treatment of RIF-1 Tumors using Selective Multiple Quantum Spectroscopy (SelMQC) and Dynamic Contrast Enhanced Imaging. NMR Biomed. 16, 102-111 (2003).

29. Bhujwalla, Z. M., Shungu, D. C., and Glickson, J. D. Effects of Blood Flow Modifiers on Tumor Metabolism Observed In Vivo by Proton Magnetic Resonance Spectroscopic Imaging. Magn. Reson. Med. 36, 204-211 (1996).

30. Less, J. R., Posner, M. C., Boucher, Y., Borochovitz, D., Wolmark, N., and Jain R. K. Interstitial Hypertension in Human Breast and Colorectal Tumors. Cancer Res. 52, 6371-6374 (1992).

31. Fischer, U., Kopka, L., and Grabbe, E. Breast Carcinoma: Effect of Preoperative Contrast-enhanced MR Imaging on the Therapeutic Approach. Radiology 213, 881-888 (1999). 
32. Nunes, L. W., Schnall, M. D., Orel, S. G., Hochman, M. G., Langlotz, C. P., Reynolds, C. A., and Torosian, M. H. Correlation of Lesion Appearance and Histologic Findings for the Nodes of a Breast MR Imaging Interpretation Model. Radiographics 19, $79-92$ (1999).

33. Nunes, L. W., Schnall, M. D., Orel, S. G., Hochman, M. G., Langlotz, C. P., Reynolds, C. A., and Torosian, M. H. Breast MR Imaging: Interpretation Model. Radiology 202, 833-841 (1997).

34. Kuhl, C. K., Mielcareck, P., Klaschik, S., Leutner, C., Wardelmann, E., Gieseke, J., and Schild, H.H. Dynamic Breast MR Imaging: Are Signal Intensity Time Course Data Dseful for Differential Diagnosis of Enhancing Lesions? Radiology 211, 101-110 (1999).

35. Ikeda, D. M., Hylton, N. M., Kinkel, K., Hochman, M. G., Kuhl, C. K., Kaiser, W. A., Weinreb, J. C., Smazal, S. F., Degani, H., Viehweg, P., Barclay, J., and Schnall, M. D. Development, Standardization, and Testing of a Lexicon for Reporting Contrastenhanced Breast Magnetic Resonance Imaging Studies. J. Magn. Reson. Imaging 13, 889-895 (2001).

36. American College of Radiology Website: http://www.acr.org/dyna/?doc=departments/stand_accred/standards/ standards.html Accessed on Sep 72004.

37. Vomweg, T. W., Teifke, A., Kunz, R. P., Hintze, C., Hlawatsch, A., Kern, A., Kreitner, K. F., and Thelen, M. Combination of Low and High Resolution Sequences in Two Orientations for Dynamic Contrast-enhanced MRI of the Breast: More than a Compromise. Eur. Radiol. 14, 1732-1742 (2004).

38. Liberman, L., Morris, E. A., Lee, M. J., Kaplan, J. B., LaTrenta, L. R., Menell, J. H., Abramson, A. F., Dashnaw, S. M., Ballon, D. J., and Dershaw, D. D. Breast Lesions Detected on MR Imaging: Features and Positive Predictive Value. Am. J. Roentgenol. 179, 171178 (2002).

39. Schnall, M. D., Rosten, S., Englander, S., Orel, S. G., and Nunes, L. W. A Combined Architectural and Kinetic Interpretation Model for Breast MR Images. Acad. Radiol. 8, 591-597 (2001).

40. Sickles, E. A. Periodic Mammographic Follow-up of Probably Benign Lesions: Results in 3,184 Consecutive Cases. Radiology 179, 463-468 (1991).

41. Kim, S. J., Morris, E. A., Liberman, L., Ballon, D. J., La Trenta, L. R., Hadar, O., Abramson, A., and Dershaw, D. D. Observer Variability and Applicability of BI-RADS Terminology for Breast MR Imaging: Invasive Carcinomas as Focal Masses. Am. J. Roentgenol. 177, 551-557(2001).

42. Rieber, A., Merkle, E., Bohm, W., Brambs, H. J., and Tomczak, R. MRI of Histologically Confirmed Mammary Carcinoma: Clinical Relevance of Diagnostic Procedures for Detection of Multifocal or Contralateral Secondary Carcinoma. J. Comput. Assist. Tomogr. 21, 773-779 (1997).

43. Slanetz, P. J., Edmister, W. B., Yeh, E. D., Talele, A. C., and Kopans, D. B. Occult Contralateral Breast Carcinoma Incidentally Detected by Breast Magnetic Resonance Imaging. The Breast Journal 8, 145 (2002).

44. Drew, P. J., Chatterjee, S., Turnbull, L. W., Read, J., Carleton, P. J., Fox, J. N., Monson, J. R., and Kerin, M. J. Dynamic Contrast Enhanced Magnetic Resonance Imaging of the Breast is Superior to Triple Assessment for the Pre-operative Detection of Multifocal Breast Cancer. Ann. Surg. Oncol. 6, 599-603 (1999).

45. Tillman, G. F., Orel, S. G., Schnall, M. D., Schultz, D. J., Tan, J. E., and Solin, L. J. Effect of Breast Magnetic Resonance Imaging on the Clinical Management of Women with Early-stage Breast Carcinoma. J. Clin. Oncol. 20, 3413-3423 (2002).

46. Bedrosian, I., Mick, R., Orel, S. G., Schnall, M., Reynolds, C., Spitz, F. R., Callans, L. S., Buzby, G. P., Rosato, E. F., Fraker, D. L., and Czerniecki, B. J. Changes in the Surgical Management of Patients with Breast Carcinoma Based on Preoperative Magnetic Resonance Imaging. Cancer 98, 468-473 (2003).
47. Hlawatsch, A., Teifke, A., Schmidt, M., and Thelen, M. Preoperative Assessment of Breast Cancer: Sonography Versus MR Imaging. Am. J. Roentgenol. 179, 1493-1501 (2002).

48. Liberman, L., Morris, E. A., Kim, C. M., Kaplan, J. B., Abramson, A. F., Menell, J. H., Van Zee, K. J., and Dershaw, D. D. MR Imaging Findings in the Contralateral Breast of Women with Recently Diagnosed Breast Cancer. Am. J. Roentgenol. 180, 333-341 (2003).

49. Hata, T., Takahashi, H., Watanabe, K., Takahashi, M., Taguchi, K., Itoh, T., and Todo, S. Magnetic Resonance Imaging for Preoperative Evaluation of Breast Cancer: A Comparative Study with Mammography and Ultrasonography. J. Am. Coll. Surg. 198, 190197 (2004).

50. Morrow, M. Magnetic Resonance Imaging in the Preoperative Evaluation of Breast Cancer: Primum non nocere. J. Am. Coll. Surg. 198, 240-241 (2004).

51. Hollingsworth, A. B. Perspectives on Preoperative Staging with Breast MRI. J. Am. Coll. Surg. 199, 173-174 (2004).

52. Egan, R. L. Multicentric Breast Carcinomas: Clinical-radiographicpathologic Whole Organ Studies and 10-year Survival. Cancer. 49, 1123-1130 (1982).

53. Vaidya, J. S., Tobias, J. S., Baum, M., Keshtgar, M., Joseph, D., Wenz, F., Houghton, J., Saunders, C., Corica, T., D’Souza, D., Sainsbury, R., Massarut, S., Taylor, I., and Hilaris, B. Intraoperative Radiotherapy for Breast Cancer. Lancet Oncol. 5, 165-173 (2004).

54. Bartelink, H. Intraoperative Radiotherapy for Breast Cancer: Tail Wagging the Dog? Lancet Oncol. 5, 207-208 (2004).

55. Fisher, E. R., Anderson, S., Tan-Chiu, E., Fisher, B., Eaton, L., and Wolmark, N. Fifteen-year Prognostic Discriminants for Invasive Breast Carcinoma: National Surgical Adjuvant Breast and Bowel Project Protocol-06. Cancer 91 (8 Suppl), 1679-1687 (2001).

56. Cuncins-Hearn, A., Saunders, C., Walsh, D., Borg, M., Buckingham, J., Frizelle, F., and Maddern, G. A Systematic Review of Intraoperative Radiotherapy in Early Breast Cancer. Breast Cancer Res. Treat. 85, 271-280 (2004).

57. Kvistad, K. A., Rydland, J., Smethurst, H. B., Lundgren, S., Fjosne, H. E., and Haraldseth, O. Axillary Lymph Node Metastases in Breast Cancer: Preoperative Detection with Dynamic Contrast-enhanced MRI. Eur. Radiol. 10, 1464-1471 (2000).

58. Silverstein, M. J., Skinner, K. A., and Lomis, T. J. Predicting Axillary Nodal Positivity in 2282 Patients with Breast Carcinoma. World J. Surg. 25, 767-772 (2001).

59. Stavros, A. T. Evaluation of Regional Lymph Nodes in Breast Cancer Patients, In Breast Ultrasound, pp. 834-876. Ed. Stavros, A. T. Lippincott Williams \& Wilkins, Philadelphia, PA (2004).

60. Luciani, A., Dao, T. H., Lapeyre, M., Schwarzinger, M., Debaecque, C., Lantieri, L., Revelon, G., Bouanane, M., Kobeiter, H., and Rahmouni, A. Simultaneous Bilateral Breast and High-resolution Axillary MRI of Patients with Breast Cancer: Preliminary Results. Am. J. Roentgenol. 182, 1059-1067 (2004).

61. Yeung, D. K., Yang, W. T., and Tse, G. M. Breast Cancer: In Vivo Proton MR Spectroscopy in the Characterization of Histopathologic Subtypes and Preliminary Observations in Axillary Node Metastases. Radiology 225, 190-197 (2002).

62. Michel, S. C., Keller, T. M., Frohlich, J. M., Fink, D., Caduff, R., Seifert, B., Marincek, B., and Kubik-Huch, R. A. Preoperative Breast Cancer Staging: MR Imaging of the Axilla with Ultrasmall Superparamagnetic Iron Oxide Enhancement. Radiology 225, 527536 (2002).

63. Yamagami, T., Yuen, S., Sawai, K., and Nishimura T. MR Imagingguided Axillary Node Biopsy for Breast Cancer: Initial Findings. Eur. Radiol. 14, 151-156 (2004).

64. Rieber, A., Zeitler, H., Rosenthal, H., Gorich, J., Kreienberg, R., Brambs, H. J., and Tomczak, R. MRI of Breast Cancer: Influence of Chemotherapy on Sensitivity. Br. J. Radiol. 70, 452-458 (1997). 
65. Ikeda, T., Jinno, H., Matsu, A., Masamura, S., and Kitajima, M. The Role of Neoadjuvant Chemotherapy for Breast Cancer Treatment. Breast Cancer 9, 8-14 (2002).

66. Londero, V., Bazzocchi, M., Del Frate, C., Puglisi, F., Di Loreto, C., Francescutti, G., and Zuiani, C. Locally Advanced Breast Cancer: Comparison of Mammography, Sonography and MR Imaging in Evaluation of Residual Disease in Women Receiving Neoadjuvant Chemotherapy. Eur. Radiol. 14, 1371-1379 (2004).

67. Partridge, S. C., Gibbs, J. E., Lu, Y., Esserman, L. J., Sudilovsky, D., and Hylton, N. M. Accuracy of MR Imaging for Revealing Residual Breast Cancer in Patients Who Have Undergone Neoadjuvant Chemotherapy. Am. J. Roentgenol. 179, 1193-1199(2002).

68. Wasser, K., Klein, S. K., Fink, C., Junkermann, H., Sinn, H. P., Zuna, I., Knopp, M. V., and Delorme, S. Evaluation of Neoadjuvant Chemotherapeutic Response of Breast Cancer Using Dynamic MRI with High Temporal Resolution. Eur. Radiol. 13, 80-87 (2002).

69. Rosen, E. L., Blackwell, K. L., Baker, J. A., Soo, M. S., Bentley, R. C., Yu, D., Samulski, T. V., and Dewhirst, M. W. Accuracy of MRI in the Detection of Residual Breast Cancer after Neoadjuvant Chemotherapy. Am. J. Roentgenol. 181, 1275-1282 (2003).

70. Martincich, L., Montemurro, F., De Rosa, G., Marra, V., Ponzone, R., Cirillo, S., Gatti, M., Biglia, N., Sarotto, I., Sismondi, P., Regge, D., and Aglietta, M. Monitoring Response to Primary Chemotherapy in Breast Cancer using Dynamic Contrast-enhanced Magnetic Resonance Imaging. Breas. Cancer Res. Treat. 83, $67-76$ (2004).

71. Fisher, E. R., Dignam, J., Tan-Chiu, E., Costantino, J., Fisher, B., Paik, S., and Wolmark, N. Pathologic Findings from the National Surgical Adjuvant Breast Project (NSABP) Eight-year Update of Protocol B-17: Intraductal Carcinoma. Cancer 86, 429-438 (1999).

72. Fisher, B., Redmond, C., Poisson, R., Margolese, R., Wolmark, N., Wickerham, L., Fisher, E., Deutsch, M., Caplan, R., and Pilch, Y. Eight-year Results of a Randomized Clinical Trial Comparing Total Mastectomy and Lumpectomy with or without Irradiation in the Treatment of Breast Cancer. N. Engl. J. Med. 320, 822-828 (1989).

73. Orel, S. G., Reynolds, C., Schnall, M. D., Solin, L. J., Fraker, D. L., and Sullivan, D.C. Breast Carcinoma: MR Imaging Before Re-excisional Biopsy. Radiology 205, 429-436 (1997).

74. Frei, K. A., Kinkel, K., Bonel, H. M., Lu, Y., Esserman, L. J., Hylton, N. M. MR Imaging of the Breast in Patients with Positive Margins after Lumpectomy: Influence of the Time Interval between Lumpectomy and MR Imaging. Am. J. Roentgenol. 175, 1577-1584 (2000).

75. Soderstrom, C. E., Harms, S. E., Farrell, R. S. Jr, Pruneda, J. M., and Flamig, D. P. Detection with MR Imaging of Residual Tumor in the Breast Soon after Surgery. Am. J. Roentgenol. 168, 485488 (1997).

76. Heywang-Kobrunner, S. H., Schlegel, A., Beck, R., Wendt, T., Kellner, W., Lommatzsch, B., Untch, M., Nathrath, W. B. Contrastenhanced MRI of the Breast after Limited Surgery and Radiation Therapy. J. Comput. Assist. Tomogr. 17, 891-900 (1993).

77. Drew, P. J., Kerin, M. J., Turnbull, L. W., Imrie, M., Carleton, P. J., Fox, J. N., and Monson, J. R. Routine Screening for Local Recurrence Following Breast-conserving Therapy for Cancer with Dynamic Contrast-enhanced Magnetic Resonance Imaging of the Breast. Ann. Surg. Oncol. 5, 265-270 (1998).

78. Rieber, A., Merkle, E., Zeitler, H., Gorich, J., Kreienberg, R., Brambs, H. J., and Tomczak, R. Value of MR Mammography in the Detection and Exclusion of Recurrent Breast Carcinoma. J. Comput. Assist. Tomogr. 21, 780-784 (1997).

79. Stuhrmann, M., Aronius, R., and Schietzel, M. Tumor Vascularity of Breast Lesions: Potentials and Limits of Contrast-enhanced Doppler Sonography. Am. J. Roentgenol. 175, 1585-1589 (2000).

80. Helbich, T. H., Matzek, W., and Fuchsjager, M. H. Stereotactic and Ultrasound-guided Breast Biopsy. Eur. Radiol. 14, 383-393 (2003).
81. Daniel, B. L., Birdwell, R. L., Butts, K., Nowels, K. W., Ikeda, D. M., Heiss, S. G., Cooper, C. R., Jeffrey, S. S., Dirbas, F. M., and Herfkens, R. J. Freehand iMRI-guided Large-gauge Core Needle Biopsy: A New Minimally Invasive Technique for Diagnosis of Enhancing Breast Lesions. J. Magn. Reson. Imaging 13, 896-902 (2001).

82. Heywang-Kobrunner, S. H., Heinig, A., Pickuth, D., Alberich, T., and Spielmann, R. P. Interventional MRI of the Breast: Lesion Localisation and Biopsy. Eur. Radiol. 10, 36-45 (2000).

83. Viehweg, P., Heinig, A., Amaya, B., Alberich, T., Laniado, M., Heywang-Kobrunner, S. H. MR-guided Interventional Breast Procedures Considering Vacuum Biopsy in Particular. Eur. J. Radiol. 42, 32-39 (2002).

84. Morris, E. A., Liberman, L., Dershaw, D. D., Kaplan, J. B., LaTrenta, L. R., Abramson, A. F., and Ballon, D. J. Preoperative, MR Imaging-guided Needle Localization of Breast Lesions. Am. J. Roentgenol. 178, 1211-1220 (2002).

85. Orel, S. G., Schnall, M. D., Newman, R. W., Powell, C. M., Torosian, M. H., and Rosato, E. F. MR Imaging-guided Localization and Biopsy of Breast Lesions: Initial Experience. Radiology 193, $97-$ 102 (1994)

86. Orel, S. G., Schnall, M. D., Newman, R. W., Powell, C. M., Torosian, M. H., and Rosato, E. F. MR Imaging-guided Localization and Liopsy of Breast Lesions: Initial Experience. Radiology 193, 97102 (1994).

87. Daniel, B. L., Birdwell, R. L., Ikeda, D. M., Jeffrey, S. S., Black, J. W., Block, W.,F., Sawyer-Glover, A. M., Glover, G. H., and Herfkens, R. J. Breast Lesion Localization: A Freehand, Interactive MR Imaging-guided Technique. Radiology 207, 455-463 (1998).

88. Prat, X., Sittek, H., Grosse, A., Baath, L., Perlet, C., Alberich, T., Lamarque, J. M., Andersson, I., Reiser, M., Taourel, P., Fischer, H., and Heywang-Kobrunner, S. H. European Quadricentric Evaluation of a Breast MR Biopsy and Localization Device: Technical Improvements Based on Phase-I Evaluation. Eur. Radiol. 12, 1720-1727 (2002).

89. Kuhl, C. K., Elevelt, A., Leutner, C. C., Gieseke, J., Pakos, E., Schild, H. H. Interventional Breast MR Imaging: Clinical Use of a Stereotactic Localization and Biopsy Device. Radiology 204, 667675 (1997).

90. Hefler, L., Casselman, J., Amaya, B., Heinig, A., Alberich, T., Koelbl, H., and Heywang-Kobrunner, S. H. Follow-up of Breast Lesions Detected by MRI not Biopsied due to Absent Enhancement of Contrast Medium. Eur. Radiol. 13, 344-346 (2003).

91. Chen, X., Lehman, C. D., and Dee, K. E. MRI-guided Breast Biopsy: Clinical Experience with 14-gauge Stainless Steel Core Biopsy Needle. Am. J. Roentgenol. 182, 1075-1080 (2004).

92. Kuhl, C. K., Morakkabati, N., Leutner, C. C., Schmiedel, A., Wardelmann, E., and Schild, H. H. MR Imaging-guided Large-core (14-gauge) Needle Biopsy of Small Lesions Visible at Breast MR Imaging Alone. Radiology 220, 31-39 (2001).

93. Perlet, C., Schneider, P., Amaya, B., Grosse, A., Sittek, H., Reiser, M. F., and Heywang-Kobrunner, S. H. MR-guided Vacuum Biopsy of 206 Contrast-enhancing Breast Lesions. Rofo. Fortschr. Geb. Rontgenstr. 174, 88-95 (2002).

94. Liberman, L., Morris, E. A., Dershaw, D. D., Thornton, C. M., Van Zee, K. J., and Tan, L. K. Fast MRI-guided Vacuum-assisted Breast Biopsy: Initial Experience. Am. J. Roentgenol. 181, 1283-1293 (2003).

95. Huber, P. E., Jenne, J. W., Rastert, R., Simiantonakis, I., Sinn, H. P., Strittmatter, H. J., von Fournier, D., Wannenmacher, M. F., and Debus, J. A New Noninvasive Approach in Breast Cancer Therapy Using Magnetic Resonance Imaging-guided Fcused Ultrasound Surgery. Cancer Res. 61, 8441-8447 (2001).

96. Gianfelice, D., Khiat, A., Amara, M., Belblidia, A., and Boulanger, Y. MR Imaging-guided Focused Ultrasound Surgery of Breast Cancer: Correlation of Dynamic Contrast-enhanced MRI with Histopathologic Findings. Breast Cancer Res. Treat. 82, 93-101 (2003). 
97. Lukas, L., Devos, A., Suykens, J. A., Vanhamme, L., Howe, F. A., Majos, C., Moreno-Torres, A., Van der Graaf, M., Tate, A. R., Arus, C., and Van Huffel, S. Brain Tumor Classification Based on Long Echo Proton MRS Signals. Artif. Intell. Med. 31, 73-89 (2004).

98. Devos, A., Lukas, L., Suykens, J. A., Vanhamme, L., Tate, A. R., Howe, F. A., Majos, C., Moreno-Torres, A., van der Graaf, M., Arus, C., and Van Huffel, S. Classification of Brain Tumours Using Short Echo Time ${ }^{1} \mathrm{H}$ MR Spectra. J. Magn. Reson. 170, 164-175 (2004).

99. Katz-Brull, R., Lavin, P. T., and Lenkinski, R. E. Clinical Utility of Proton Magnetic Resonance Spectroscopy in Characterizing Breast Lesions. J. Natl. Cancer Inst. 94, 1197-1203 (2002).

100. Cousins, J. P. Clinical MR Spectroscopy: Fundamentals, Current Applications, and Future Potential. Am. J. Roentgenol. 164, 13371347 (1995).

101. Yeh, E. D. Characterization of Breast Lesions with Proton MR Spectroscopy. Am. J .Roentgenol. 181, 1273-1274 (2003).

102. Yeung, D. K., Cheung, H. S., Tse, G. M. Human Breast Lesions: Characterization with Contrast-enhanced In Vivo Proton MR Spectroscopy - Initial Results. Radiology 220, 40-46 (2001).

103. Thomas, M. A., Binesh, N., Yue, K., and DeBruhl, N. Volume-localized Two-dimensional Correlated Magnetic Resonance Spectroscopy of Human Breast Cancer. J. Magn. Reson. Imaging. 14, 181186 (2001).

104. Cecil, K. M., Schnall, M. D., Siegelman, E. S., and Lenkinski, R. E. The Evaluation of Human Breast Lesions with Magnetic Resonance Imaging and Proton Magnetic Resonance Spectroscopy. Breast Cancer Res. Treat. 68, 45-54 (2001).

105. Tse, G. M., Cheung, H. S., Pang, L. M., Chu, W. C., Law, B. K., Kung, F. Y., and Yeung, D. K. Characterization of Lesions of the Breast with Proton MR Spectroscopy: Comparison of Carcinomas, Benign Lesions, and Phyllodes Tumors. Am. J. Roentgenol. 181, 1267-1272 (2003).

106. Jacobs, M. A., Barker, P. B., Bottomley, P. A., Bhujwalla, Z., and Bluemke, D. A. Proton Magnetic Resonance Spectroscopic Imaging of Human Breast Cancer: A Preliminary Study. J. Magn. Reson. Imaging 19, 68-75 (2004).

107. Huang, W., Fisher, P. R., Dulaimy, K., Tudorica, L. A., O’Hea, B., and Button, T. M. Detection of Breast Malignancy: Diagnostic MR Protocol for Improved Specificity. Radiology 232, 585-591 (2004).

108. Kvistad, K. A., Rydland, J., Vainio, J., Smethurst, H. B., Lundgren, S., Fjosne, H. E., and Haraldseth, O. Breast Lesions: Evaluation with Dynamic Contrast-enhanced T1-weighted MR Imaging and with T2*-weighted First-pass Perfusion MR Imaging. Radiology 216, 545-553 (2000).

109. Duffy, S. W., Tabar, L., Chen, H. H., Holmqvist, M., Yen, M. F., Abdsalah, S., Epstein, B., Frodis, E., Ljungberg, E., HedborgMelander, C., Sundbom, A., Tholin, M., Wiege, M., Akerlund, A., Wu, H. M., Tung, T. S., Chiu, Y. H., Chiu, C. P., Huang, C. C., Smith, R. A., Rosen, M., Stenbeck, M., and Holmberg, L. The Impact of Organized Mammography Service Screening on Breast Carcinoma Mortality in Seven Swedish Counties. Cancer 95, 458-469 (2002).

110. Kopans, D. B. Updated Results of the Trials of Screening Mammography. Surg. Oncol. Clin. N. Am. 6, 233-263 (1997).
111. Nystrom, L., Andersson, I., Bjurstam, N., Frisell, J., Nordenskjold, B., and Rutqvist, L. E. Long-term Effects of Mammography Screening: Updated Overview of the Swedish Randomised Trials. Lancet 359, 909-919 (2002).

112. Kopans, D. B. The Most Recent Breast Cancer Screening Controversy about Whether Mammographic Screening Benefits Women at Any Age: Nonsense and Nonscience. Am. J. Roentgenol. 180, 21-26 (2003).

113. Irwig, L., Houssami, N., and van Vliet, C. New Technologies in Screening for Breast Cancer: A Systematic Review of Their Accuracy. Br. J. Cancer 90, 2118-2122 (2004).

114. Morris, E. A., Liberman, L., Ballon, D. J., Robson, M., Abramson, A. F., Heerdt, A., and Dershaw, D. D. MRI of Occult Breast Ccarcinoma in a High-risk Population. Am. J. Roentgenol. 181, 619626 (2003).

115. Ford, D. and Easton, D. F. The Genetics of Breast and Ovarian Cancer. Br. J. Cancer 72, 805-812 (1995).

116. Kuhl, C. K., Schmutzler, R. K., Leutner, C. C., Kempe, A., Wardelmann, E., Hocke, A., Maringa, M., Pfeifer, U., Krebs, D., and Schild, H. H. Breast MR Imaging Screening in 192 Women Proved or Suspected to be Carriers of a Breast Cancer Susceptibility Gene: Preliminary Results. Radiology 215, 267-279 (2000).

117. Kerlikowske, K., Carney, P. A., Geller, B., Mandelson, M. T., Taplin, S. H., Malvin, K., Ernster, V., Urban, N., Cutter, G., Rosenberg, R., and Ballard-Barbash, R. Performance of Screening Mammography Among Women With and Without a First-degree Relative With Breast Cancer. Ann. Intern. Med. 133, 855-863 (2000).

118. Lakhani, S. R., Jacquemier, J., Sloane, J. P., Gusterson, B. A., Anderson, T. J., van de Vijver, M. J., Farid, L. M., Venter, D., Antoniou, A., Storfer-Isser, A., Smyth, E., Steel, C. M., Haites, N., Scott, R. J., Goldgar, D., Neuhausen, S., Daly, P. A., Ormiston, W., McManus, R., Scherneck, S., Ponder, B. A., Ford, D., Peto, J., Stoppa-Lyonnet, D., and Easton, D. F. Multifactorial Analysis of Differences between Sporadic Breast Cancers and Cancers Involving BRCA1 and BRCA2 Mutations. J. Natl. Cancer Inst. 90, 1138-1145 (1998).

119. Burke, W., Daly, M., Garber, J., Botkin, J., Kahn, M. J., Lynch, P., McTiernan, A., Offit, K., Perlman, J., Petersen, G., Thomson, E., and Varricchio, C. Recommendations for Follow-up Care of Individuals with an Inherited Predisposition to Cancer. II. BRCA1 and BRCA2. Cancer Genetics Studies Consortium. J.A.M.A. 277, 997-1003 (1997).

120. Goscin, C. P., Berman, C. G., and Clark, R. A. Magnetic Resonance Imaging of the Breast. Cancer Control 8, 399-406 (2001).

121. Kriege, M., Brekelmans, C. T., Boetes, C., Besnard, P. E., Zonderland, H. M., Obdeijn, I. M., Manoliu, R. A., Kok, T., Peterse, H., Tilanus-Linthorst, M. M., Muller, S. H., Meijer, S., Oosterwijk, J. C, Beex, L. V., Tollenaar, R. A., de Koning, H. J., Rutgers, E. J., and Klijn, J. G. Magnetic Resonance Imaging Screening Study Group. Efficacy of MRI and Mammography for Breast-cancer Screening in Women with a Familial or Genetic Predisposition. $N$. Engl. J. Med. 351, 427-437 (2004).

Date Received: September 28, 2004 